\title{
RETRACTED ARTICLE: Determining the Sites of Neural Adaptations to Resistance Training: A Systematic Review and Meta-Analysis
}

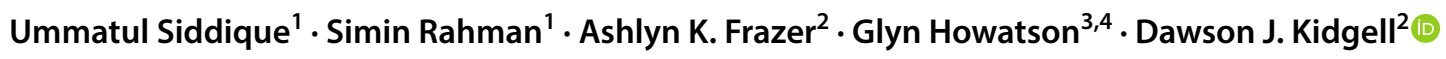

Published online: 29 July 2019

(c) Springer Nature Switzerland AG 2019

The authors have retracted this article [1] because after publication it was brought to their attention that the statistical approach is not appropriate. The authors are re-analysing their data and intend to submit a new manuscript for peer review in due course. All authors agree to this retraction.
[1] Siddique U, Rahman S, Frazer AK, Howatson G, Kidgell D. Sports Med (2019). https://doi.org/10.1007/s4027 9-019-01152-3

Electronic supplementary material The online version of this article (https://doi.org/10.1007/s40279-019-01152-3) contains supplementary material, which is available to authorized users.

Dawson J. Kidgell

dawson.kidgell@monash.edu

1 Department of Neurology, Institute of Neurosciences, Kolkata, India

2 Department of Physiotherapy, School of Primary and Allied Health Care, Faculty of Medicine, Nursing and Health Science, Monash University, PO Box 527, Frankston, VIC 3199, Australia

3 Faculty of Health and Life Sciences, Northumbria University, Newcastle-upon-Tyne, UK

4 Water Research Group, School of Environmental Sciences and Development, Northwest University, Potchefstroom, South Africa 